\title{
Motion control of a cable-restricted underwater vehicle for long-term spot observation
}

\author{
Yoshiki Tanaka \\ Dept. of Life Science and Systems Engineering, Kyushu Institute of Technology, 2-4 Hibikino, Wakamatsu-ku, \\ Kitakyushu, Fukuoka, 808-0196, Japan \\ Yuya Nishida \\ Dept. of Human Intelligence Systems, Kyushu Institute of Technology, 2-4 Hibikino, Wakamatsu-ku, Kitakyushu, \\ Fukuoka, 808-0196, Japan \\ Kazuo Ishii \\ Dept. of Human Intelligence Systems, Kyushu Institute of Technology, 2-4 Hibikino, Wakamatsu-ku, Kitakyushu, \\ Fukuoka, 808-0196, Japan \\ E-mail: tanaka.yoshiki732@mail.kyutech.jp,y-nishida@brain.kyutech.ac.jp, ishii@brain.kyutech.ac.jp
}

\begin{abstract}
In order to acquire a time fluctuation data of the resources which needs for a marine resource development, we developed an observation device with low operational risk and a wide observable area. The observation device consists of an underwater station and an underwater vehicle, and underwater vehicle is tethered with a cable. By using the restraint condition of the cable, our device was able to navigate the trajectory planned only by thrust control with an error of up to $0.14 \mathrm{~m}$.
\end{abstract}

Keywords: Underwater vehicle, Long-term observation, Cable-tethered, Self-localization

\section{Introduction}

In Japan's exclusive economic zone, there are many marine resources including mineral resources and energy resources [1]. These are very important basic resources for the development of an industrial society, but they have not yet reached the economic use of marine resources. The reason is that these resources are widly distributed on the seabed at a depth of $50 \mathrm{~m}$ or more where general divers cannot dive, it is difficult for divers to search and observation. Un-tethered underwater vehicles, AUVs (automonous underwater vehicles) are used as the practical tools to investigate these marine resources [2][3]. However, the AUVs have the risk of their losing itself on a hardware or software failures, the AUVs cannot be used often for the resource investigation. Thus, a time fluctuation data of the resources which needs for a marine resource development cannot be obtained by an existing survey. This research aims to develop a longterm observation device with low operation risk and wide observable area to acquire a time fluctuation data. In this paper, we explain the proposed long-term observation device and show the experimental results for evaluating the position accuracy using the underwater vehicle.

\section{Trajectory model and Motion control}

\subsection{Overview}

As shown in Fig.1, the proposed system consists of an underwater station for supplying electric power and an underwater vehicle, the underwater vehicle is tethered to the underwater station with a cable. Underwater vehicle can observe around the underwater station for a longterm by keeping the altitude constant and navigating while applying tension to the cable. By using the restraint condition by tethering, an underwater vehicle can navigate the same trajectory with only simple motion control without using self-localization based on DVL or INS or using waypoints. Therefore, it is possible to observe a wide area with lower risk than existing underwater vehicles.

(C) The 2021 International Conference on Artificial Life and Robotics (ICAROB2021), January 21 to 24, 2021 


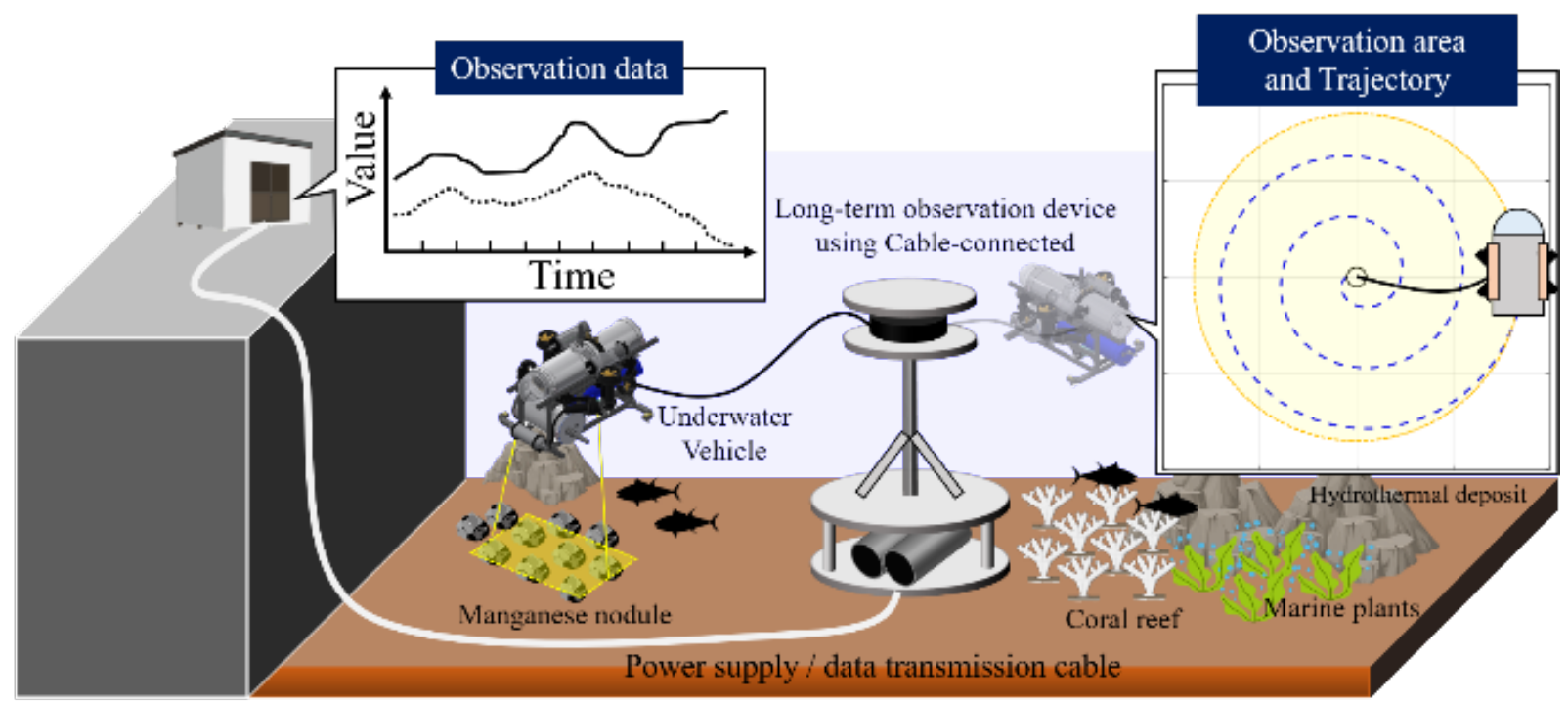

Fig. 1 Overview of proposal method

\subsection{Trajectory model}

To derive the relationship between the structure of the underwater station and the trajectory of the underwater vehicle, we constructed a theoritical formula based on the involute curve. The involute curve is a curved line by the end point $p_{i}=\left[x_{i} y_{i}\right]^{T}$ of the cable when the cable wound around the basic circular radius $a$ centered on the origin us always pulled and unwound, $x_{i}$ and $y_{i}$ are expressed by the following equations:

$$
\begin{aligned}
& x_{i}=a(\cos \theta+\theta \sin \theta) \\
& y_{i}=a(\sin \theta-\theta \cos \theta)
\end{aligned}
$$

Here, $\theta$ express the angle from the initial position. In this research, we consider that the basic circular radius $a$ changes by the cable thickness $d_{c a}$ depending on the number of times $n$ of winding and unwinding of the cable. Thus, the basic circular radius $a_{i}$ is defined by the following equations:

$$
\begin{gathered}
a_{w i}=n \cdot d_{c a}+r_{b} \\
a_{u w i}=(N-n) d_{c a}+r_{b}
\end{gathered}
$$

Here, $a_{w i}$ is the basic radius when winding the cable, $a_{u w i}$ is the basic radius when unwinding the cable, and $n$ is the maximum number of times the cable can be winding and unwinding. $r_{b}$ is the radius of the device, and we adopted a parameter that considers the allowable bending radius of the cable used. Considering that the length of the cable when winding or unwinding changes depending on the number of times $n$ and the angle $\theta$, it is defined by the following equations:

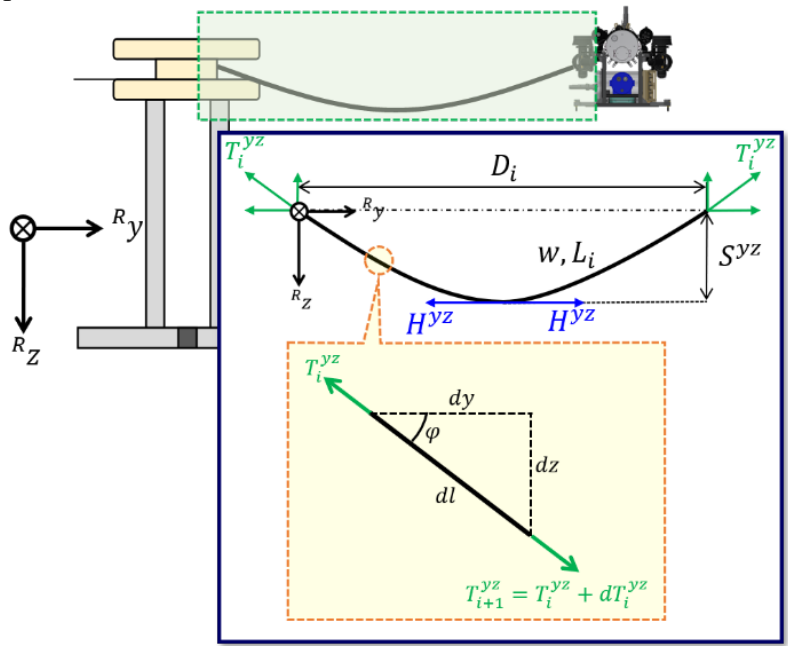

Fig. 2 Cable model

$$
\begin{aligned}
& L_{w i}=L_{m}-2 \pi\left\{n \cdot r_{b}+\frac{1}{2} n(n-1) d_{c a}\right\} \\
& L_{u w i}=2 \pi n\left[r_{b}+\left\{N-\frac{1}{2} n(n-1)\right\} d_{c a}\right]
\end{aligned}
$$

Here, $L_{w i}$ is the cable length when winding, $L_{u w i}$ is the cable length when unwinding, and $L_{m}$ is the maximum length of the cable. When applied to Eq. (1) with the changes in the radius of the device and cable length

(C) The 2021 International Conference on Artificial Life and Robotics (ICAROB2021), January 21 to 24, 2021 
calculated from Eq. (2) and Eq. (4) as constraints, the trajectory $p_{w i}=\left[x_{w i}, y_{w i}\right]^{T}$ at the time of winding is expressed by the following equation:

$$
\begin{aligned}
& x_{w i}=a_{w i} \cos \theta-\left(L_{w i}-a_{w i} \theta\right) \sin \theta \\
& y_{w i}=a_{w i} \sin \theta+\left(L_{w i}-a_{w i} \theta\right) \cos \theta
\end{aligned}
$$

Similarly, the trajectory $p_{u w i}=\left[x_{u w i}, y_{u w i}\right]^{T}$ at the time of unwinding is expressed by the following equation:

$$
x_{u w i}=a_{u w i} \cos \theta-\left(L_{u w i}+a_{u w i} \theta\right) \sin \theta
$$$$
y_{u w i}=a_{u w i} \sin \theta+\left(L_{u w i}+a_{u w i} \theta\right) \cos \theta
$$

When the cable length calculated from Eq. (4) or Eq. (5) reaches $L_{m}$, the trajectory of the underwater vehicle becomes a circle centered on the device. Therefore, it is expressed by the following equation.

$$
\begin{aligned}
& x_{i}=L_{m} \cos \theta \\
& y_{i}=L_{m} \sin \theta
\end{aligned}
$$

Here, the cable length in the trajectory calculated fromEq. (8) is symmetrical around the device. Therefore, the range of the angle $\theta$ is $0 \leq \theta \leq 180$ [deg].

\subsection{Motion control}

In the water, the weight and fluid resistance of the cable increase depending on the length of the unwound cable, so the sag of the cable increases. As the sag increases, it becomes more difficult for underwater vehicle to draw the planned trajectory and the positioning accuracy decreases. Therefore, we calculated the thrust required in the direction of travel and the thrust required in the direction of extension. At first, we explain the thrust force $F_{S R G}$ required in the direction of travel. In the future, we plan to create a map from seabed images by a camera mounted on an underwater vehicle. We are considering the creation of a seafloor map for long-term observation of marine resources. In order to create a map using images taken by the equipped camera in underwater vehicle, it is necessary to input velocity parameters to the underwater vehicle so that the captured images overlap. The velocity $V_{x}$ required to satisfy a specific overlap rate is expressed by the following equation:

$$
V_{x} \leq \frac{d_{S}\left(1-\alpha_{O L}\right)}{T_{S}}
$$

Here, $d_{S}$ is the shooting range of the camera, $\alpha_{O L}$ is the overlap rate, and $T_{S}$ is the shooting cycle. By substituting the velocity calculated from Eq. (9) into the following equation, the thrust $F_{S R G}$ in the direction of travel can be obtained.

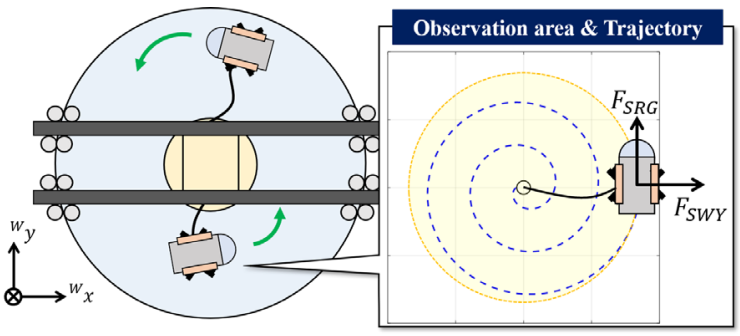

Fig.3 Experimental setup

Table1 Thrust value $F_{S R G}$ and $F_{S W Y}$

\begin{tabular}{|c|c|c|c|c|}
\hline$F_{S R G}[\mathrm{~N}]$ & \multicolumn{4}{|c|}{$F_{S W Y}[\mathrm{~N}]$} \\
\hline 4.0 & 5.0 & 10.0 & 15.0 & 20.0 \\
\hline 9.0 & 5.0 & 10.0 & 15.0 & 20.0 \\
\hline 16.0 & 5.0 & 10.0 & 15.0 & 20.0 \\
\hline
\end{tabular}

$$
F_{S R G}=D_{x}\left|V_{x}\right| V_{x}
$$

Here, $D_{x}$ is the fluid drag force. This parameter was approximated by experiment. Next, we explain the thrust force $F_{S W Y}$ in the direction of extension. Fig. 2 shows the cable model of the underwater vehicle when it is tethered to the underwater station. If the cable has ideal sag without considering the stiffness, and the underwater vehicle moves slowly, the cable can be considered to have a catenary shape [4]. Therefore, the sag $z_{c i}$ generated by the cable's own weight and the tension $T_{i}^{y z}$ applied to the cable can be expressed by the following eqations:

$$
\begin{gathered}
z_{c i}=\frac{H^{y z}}{w}\left\{\cosh \frac{w D_{m}}{2 H^{y z}}-\cosh \frac{w}{H^{y z}}\left(\frac{D_{m}}{2}-D_{i}\right)\right\} \\
T_{i}^{y z} \geq H^{y z} \cosh \frac{w}{H^{y z}}\left(\frac{D_{m}}{2}-D_{i}\right)
\end{gathered}
$$

Here, $H^{y z}$ is the horizontal tension at the lowest point of the cable, $w$ is the weight in water per unit cable length, $D_{i}$ is the distance between center of the device and underwater vehicle, $D_{m}$ is the maximum distance between center of the device and underwater vehicle. Therefore, the thrust force in the direction of extension required for the underwater vehicle to suppress the sag caused by the weight of the cable is expressed by the following equation:

$$
F_{S W Y} \geq T_{i}^{y z} \cos \varphi
$$

In this paper, we calculated the minimum thrust $F_{S W Y}$ in the direction of extension by assuming that the trajectory of the underwater vehicle obtained from Eq. (6) to Eq. (9) contains an error of at most $1 \%$ due to cable's sag.

\section{Wet test and evaluation of trajectory}

To evaluate the trajectory of the cable-tethered underwater vehicle described in section 2.2, we 


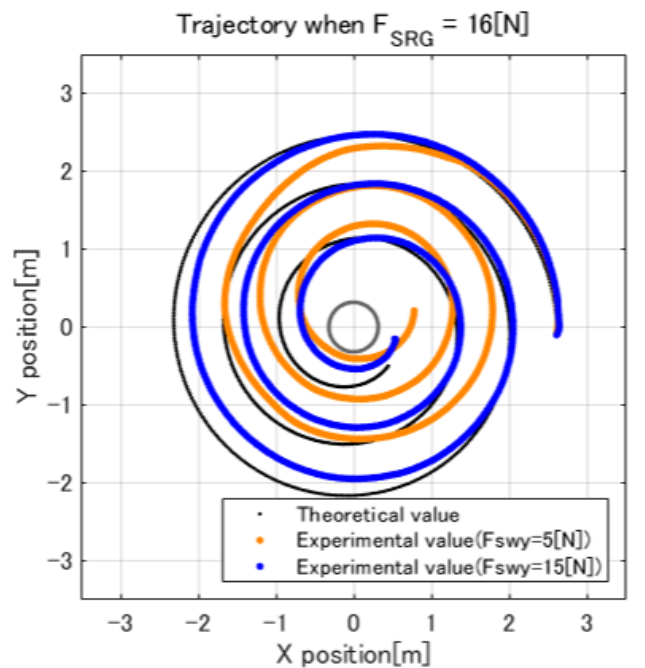

Fig.4 Trajectory of AUV KYUBIC

(Theoretical value vs. Experimental value)

experimented using the hovering type AUV KYUBIC. This AUV is equipped with IMU, DVL and depth sensors as navigation sensors. We used the velocity data from DVL and the heading data from IMU to evaluate the selflocalization. Fig. 3 shows an overview of this experiment. The AUV is tethered to the device by a cable (thickness is $12 \mathrm{~mm}$ and length is $2.2 \mathrm{~m}$ ). The device resembling an underwater station is set in the center of the water tank (diameter is $6 \mathrm{~m}$ and depth is $1.2 \mathrm{~m}$ ). In this experiment, we used the parameters shown in Table1 as the thrust force applied to the AUV and its self-localization was measured when winding the cable. Fig. 4 shows the trajectory calculated using Eq. (6) and the selflocalization of the AUV at $F_{S R G}=16[\mathrm{~N}]$. The positional accuracy is improved by increasing the thrust force $F_{S W Y}$. However, a different trajectory from the theoretical value can be confirmed immediately after the AUV starts moving. This cause is considered that the azimuth during navigation contained an error with the teoretical value because the AUV moved only by thrust control. Table2 to Table4 show the mean square error (MSE) of the selflocalization when the thrust applied to the AUV is changed. It can be confirmed that the distance error between the center of the device and AUV is reduced by increasing the thrust force $F_{S W Y}$. However, the MSE of distance at $F_{S W Y}=20[\mathrm{~N}]$ is larger than at $F_{S W Y}=15[\mathrm{~N}]$. This cause is considered that the velocity of the AUV is reduced by increasing the thrust force $F_{S W Y}$, and the position data calculated by the DVL and IMU contain accumulated error.
Table2 Position error when $F_{S R G}=4[\mathrm{~N}]$

\begin{tabular}{|c|c|c|c|}
\hline \multirow{2}{*}{$\begin{array}{c}F_{S W Y} \\
{[\mathrm{~N}]}\end{array}$} & \begin{tabular}{c}
$\mathrm{X}$ \\
\cline { 2 - 4 }
\end{tabular} & $\begin{array}{c}\mathrm{Y} \\
\text { position[m] }\end{array}$ & $\begin{array}{c}\text { Distance } \\
\text { position[m] }\end{array}$ \\
\hline 5.0 & 0.06 & 0.03 & 0.07 \\
\hline 10.0 & 0.03 & 0.03 & 0.03 \\
\hline 15.0 & 0.02 & 0.04 & 0.03 \\
\hline 20.0 & 0.01 & 0.10 & 0.06 \\
\hline
\end{tabular}

Table3 Position error when $F_{S R G}=9[\mathrm{~N}]$

\begin{tabular}{|c|c|c|c|}
\hline \multirow{2}{*}{$\begin{array}{c}F_{S W Y} \\
{[\mathrm{~N}]}\end{array}$} & $\begin{array}{c}\mathrm{X} \\
\text { position[m] }\end{array}$ & $\begin{array}{c}\text { MSE } \\
\text { position[m] }\end{array}$ & $\begin{array}{c}\text { Distance } \\
{[\mathrm{m}]}\end{array}$ \\
\hline 5.0 & 0.06 & 0.05 & 0.07 \\
\hline 10.0 & 0.05 & 0.03 & 0.04 \\
\hline 15.0 & 0.03 & 0.04 & 0.04 \\
\hline 20.0 & 0.03 & 0.11 & 0.07 \\
\hline
\end{tabular}

Table4 Position error when $F_{S R G}=16[\mathrm{~N}]$

\begin{tabular}{|c|c|c|c|}
\hline \multirow{2}{*}{$\begin{array}{c}F_{S W Y} \\
{[\mathrm{~N}]}\end{array}$} & \begin{tabular}{c}
$\mathrm{X}$ \\
\cline { 2 - 4 }
\end{tabular} & $\begin{array}{c}\mathrm{Y} \\
\text { position[m] }\end{array}$ & $\begin{array}{c}\text { Distance } \\
\text { position[m] }\end{array}$ \\
\hline 5.0 & 0.12 & 0.14 & 0.14 \\
\hline 10.0 & 0.05 & 0.07 & 0.05 \\
\hline 15.0 & 0.03 & 0.02 & 0.03 \\
\hline 20.0 & 0.03 & 0.09 & 0.06 \\
\hline
\end{tabular}

\section{Conclusions}

We proposed a new method that can observe a wide area with lower risk than exisiting method. Our instrument was able to move only by thrust control, with an average error of up to $0.14 \mathrm{~m}$ relative to the theoreticel value. In future work, we plan to evaluate the trajectory when unwinding the cable and add the heading control.

\section{References}

1. C.E. Fitzgerald, et al., Hydrothrmal Manganese Oxide Deposits from Baby Bare Seamount in the Northeast Pacific Ocean, Marine Geology, Vol.225, Issue 1-4, pp. $145-156,2006$

2. Y. Nishida, et al., Development of an autonomous underwater vehicle for survey of cobalt-rich manganese crust, OCEANS2015 MTS/IEEE Washington, pp.1-5, 2015

3. Toshihiro Maki, et al., Docking Method for HoveringType AUVs Based on Acoustic and Optical Landmarks, Journal of Robotics and Mechatronics, Vol.30, No.1, pp.55-64, 2018

4. Atsushi Imadu, et al., Control Method for Helicopters Tethered to Ground Station with Compensation for Disturbance Caused by Cable Tension, Jornal of Robotics and Mechatronics, Vol.29, No.4, pp.737-745, 2017

(C) The 2021 International Conference on Artificial Life and Robotics (ICAROB2021), January 21 to 24, 2021 